\title{
A polyhedral approach for understanding flavonoid biosynthesis in Arabidopsis
}

Ryo Nakabayashi ${ }^{1,2}$, Mami Yamazaki ${ }^{1,2}$ and Kazuki Saito ${ }^{1,3^{*}}$

${ }^{1}$ Graduate School of Pharmaceutical Sciences, Chiba University, 1-33 Yayoi-cho, Inage-ku, Chiba 263-8522, Japan

${ }^{2}$ CREST, Japan Science and Technology Agency, 4-1-8 Honcho, Kawaguchi, Saitama 332-0012, Japan

${ }^{3}$ RIKEN Plant Science Center, 1-7-22 Suehiro-cho, Tsurumi-ku, Yokohama 230-0045, Japan

*Corresponding author:

K. Saito

E-mail: ksaito@faculty.chiba-u.jp Tel.: +81-43-290-2904, Fax: +81-43-290-2905 


\begin{abstract}
The sequencing of the entire genome of model plants has made it is possible to use these plants for producing metabolites that are beneficial to humans. Plants produce a large number of metabolites, which are potentially valuable sources of novel pharmaceutically active agents that benefit human health. The industrial use of these beneficial metabolites from plants, known as secondary metabolites or specialized metabolites, necessitates the systematic understanding of the biosynthetic mechanisms from the genetic to the metabolic level. Here, we review the recent developments on flavonoid biosynthesis in Arabidopsis thaliana (Arabidopsis) as a model plant. These developments have been made in the fields of metabolomics, transcriptomics, natural products chemistry, genetics, and biochemistry. We focused on natural products chemistry in this polyhedral approach, which proved to be indispensable for elucidating the secondary metabolism in not only Arabidopsis but also other model-like and crop plants.
\end{abstract}

Keywords: Arabidopsis thaliana, metabolomics, transcriptomics, natural products chemistry, forward/reverse genetics, biochemistry, secondary metabolites, flavonoid, flavonol, anthocyanin 


\section{Introduction}

Plants produce a large number of metabolites that are an abundant source of novel pharmaceutically active agents [1]. The sequencing of the entire genome of model plants [2-8] has made it possible to use these plants for producing novel secondary metabolites, or alternatively referred as 'specialized metabolites', that are beneficial to humans [9]. For the industrial use of plants as biosynthetic machinery, we first have to understand their biosynthetic mechanisms from the genetic to the metabolic level (Fig. 1). We then need to optimize the machinery using overexpression, knockout, or knockdown methods of the targeted genes for high-producing secondary metabolites in the most efficient way $[10,11]$. Elucidating the biological roles of metabolites and the process of their evolution under various environmental conditions can likely help to increase the manufacture yield [12-15]. For the stable production of secondary metabolites, we finally need the construct of large-scale cell or hairy root cultures $[16$, 17]. Our knowledge of the biosynthetic mechanisms of secondary metabolites in Arabidopsis thaliana (Arabidopsis) and many other plants is continuously expanding at various points [18-23].

Flavonoids constitute a large group of secondary metabolites that play important roles in plants such as attracting insects, UV-B protection, phytoalexins, allelochemicals, and antioxidants [24-29]. Flavonoids are also useful for humans because of their antioxidant, antiinflammatory, anticancer, antifungal, and antiviral properties [30-34]. The biosynthesis of flavonols and anthocyanins involves the 
construction of the basic skeleton, followed by the addition of the modifying moiety. Flavonoid biosynthesis that requires metabolites and genes encoding structural enzymes, modification enzymes, and transcription factors (TFs) is still not fully understood even in Arabidopsis. This fact could be attributed to the chemical diversity of flavonoids and the complexity of the associated mechanisms [35-38].

Integrated analysis of metabolomics and transcriptomics has recently provided an improved understanding of plant secondary metabolism from the genetic to the metabolic level [39-49]. Metabolomics involves the comprehensive identification and quantification of metabolites. However, all metabolites, especially secondary metabolites, have not been identified with this method because of the lack of standard compounds. Unequivocal identification of metabolites by natural products chemistry, which provides detailed structure information on chemical compounds, is crucial for the development of metabolomics [50, 51]. Recently, a polyhedral approach has been shown to be a powerful tool for decoding gene function (functional genomics) and understanding the biosynthetic mechanisms of flavonoids [9]. The polyhedral approach encompasses omics-guided fusion approaches from the genetic to the metabolic level (Fig. 1). For example, the combination of metabolomics and natural products chemistry could be referred to as a metabolomics-guided fusion approach, whereas the combination of transcriptomics, biochemistry, and genetics could be regarded as a transcriptomics-guided fusion approach. These fusion approaches enable clear identification of metabolites and the functions of genes of interest. The polyhedral approach is required for an accurate understanding of cellular function and, hence, 
furthering the applications of metabolites in plant biotechnology.

Here, we review recent studies on the use of the polyhedral approach including natural products chemistry for comprehensively understanding the flavonoid biosynthesis. 


\section{Metabolomics and natural products chemistry combination approach to identify flavonoids in Arabidopsis}

Targeted analyses of flavonoids in Arabidopsis have previously been conducted [41, 42, 52]. Flavonoid-targeted analysis by high performance liquid chromatography-mass spectrometry (LC-MS) was conducted using samples collected from different plant organs and extracted with a specific solvent, $\mathrm{MeOH}-\mathrm{H}_{2} \mathrm{O}-\mathrm{CH}_{3} \mathrm{COOH}$ (9:10:1). Extraction from each organ (flower, leaf, stem, and root) yielded a higher flavonoid concentration than extraction from whole plants and resulted in a high detection rate in LC-MS analysis. Flavonoid-targeted analysis by LC-MS has resulted in the detection of 32 flavonols and 11 anthocyanins derived from 4 aglycones namely kaempferol, quercetin, isorhamnetin, and cyanidine. This method has revealed their distribution in each organ (flower, leaf, stem, and root) in a wild-type plant (Col-0) and in a mutant (pap 1-D) overexpressing an MYB transcription factor (Table 1). Through the use of this analysis along with flavonoid-defective mutants, the positions at which hydroxylation, methylation, and glycosylation occur were identified. In addition, all the detected flavonoids were examined for their retention time, possible fragment ions, UV spectrum, and presence/absence in mutants [41, 42, 53-55].

The predicted structures of flavonoids have been confirmed by metabolomics-oriented isolation and structure elucidation [52]. Seven flavonols (f1, $\mathrm{f} 2$, f3, f5, f6, f8, and f24) and 7 anthocyanins (A3, A5, A6, A8, A9, A10, and A11) have been identified using authentic standards isolated from Arabidopsis (Table 1). After the 
fragment ions, retention time, and UV spectrum were annotated using LC-MS, new structures (A8 and A10) were elucidated using the approaches of natural products chemistry. Further, biosynthetic pathways were delineated on the basis of previous research wherein the structures of flavonoids and novel isolated compounds were identified by targeted analysis with flavonol-defective mutants. After obtaining accurate structural information was obtained, a possible biosynthetic pathway from cyanidin to A11, which can be referred to as the "Anthocyanin biosynthetic grid of Arabidopsis" was proposed. This pathway involves acylation and glycosylation reactions (Fig. 2) [56, 57].

The exact information from the combination of metabolomics and natural products chemistry is useful for narrowing down candidate genes in the conduct of functional genomics [51]. For example, organ specific flavonoid with a new sugar likely indicates the existence of a new gene encoding glycosyltransferase and the expression part of the gene. The gene expression should be done at the almost same part where the flavonoid is accumulated. This focusing to metabolite information, metabolite accumulation, and gene expression will be surely applied to identification of other unknown genes involved in flavonoid biosynthetic pathway. 


\section{Natural products chemistry and biochemistry combination approach to identify the function of flavonoid-related genes in Arabidopsis}

Flavonoid can be biosynthesized via the conversion of aglycone to glycoside by the action of genes involved in early and late biosynthesis [58]. Genes involved in early and late biosynthetic processes, ie., CHS/tt4, CHI/tt5, F3H/tt6, F3'H/tt7, DFR/tt3, and ANS/tt15, which encode biosynthetic enzymes from $p$-coumarate to flavonol and anthocyanin aglycones, are well known. On the other hand, the function of genes encoding enzymes involved in the glycosylation of flavonols is only partially understood, even in Arabidopsis. In fact, the primary biosynthetic pathway in the Anthocyanin biosynthetic grid of Arabidopsis is also ambiguous. This biosynthetic pathway is thought to be controlled by MYB TFs together with the proteins bHLH and WD40. Recently, several flavonoid-related MYB TFs have been characterized. MYB11/PFG2, MYB12/PFG1, and MYB111/PFG3 regulate flavonol biosynthesis, and MYB75/PAP1, MYB90/PAP2, MYB113, MYB114, and MYBL2 regulate anthocyanin biosynthesis. Each TF rigidly and independently regulates the expression of target genes [59-64] (Fig. 2).

Metabolite biosynthesis generally occurs in coordination with the expression of genes involved in the biosynthesis of individual metabolites at individual organs. Thus, a large amount of information for mining genes involved in the biosynthesis of secondary metabolites can be obtained from DNA microarray data, which depict snapshots of gene expression at a certain point in time and an organ $[65,66]$. The 
database ATTED-II [67] provides coexpression sets of microarray data collected according to the developmental stage, stress induced, and hormone treatment given to the plants [68]. Using known genes as guide-genes proved useful for predicting new candidate genes - not only biosynthetic genes but also genes encoding TFs involved in flavonoid biosynthesis (guide-gene approach) [69]. Transcriptome coexpression analysis in ATTED-II was performed using known flavonoid-related genes that encode biosynthetic enzymes or TFs and 107 UDP glycosyltransferase (UGT) genes in order to identify new candidate genes that encode flavonol glycosyltransferase [41, 55].

Natural products chemistry has shown the importance of characterizing the functions of genes that encode anthocyanin 5-O-glucoside:6"'-O-malonyltransferase in the grid as well as flavonol 3-O-arabinosyltransferase (F3AraT/UGT78D3) and flavonol 7-O-rhamnosyltransferase (F7RhaT/UGT89C1) in the flavonoid-glycoside biosynthetic pathway (Fig. 2) [41, 55, 57]. Using natural products chemistry and biochemistry, Luo et al. [57] found that the most efficient reaction of malonyltransferase was via the conversion of $\mathrm{A} 3$ to $\mathrm{A} 5$ as well as bypaths from $\mathrm{A} 7$ to $\mathrm{A} 9$ and $\mathrm{A} 6$ to $\mathrm{A} 8$ by using in vitro recombinant proteins and standard compounds. In addition, these approaches suggest that the end products of Arabidopsis anthocyanin are A10 and A11. Through conventional approaches for the characterization of UGT78D3 as F3AraT, 2 flavonols (f19 and f25) were synthesized by the enzymatic reaction of F7RhaT with kaempferol 3-O-arabinoside and quercetin 3-O-arabinoside and identified by the approaches of natural products chemistry, because these 2 flavonols (f19 and f25) were not commercially available (Table 1) [41]. Another flavonol (f1) also helped to characterize 
the function of UGT89C1 as F7RhaT and led to the discovery of a new biosynthetic pathway [55].

It is difficult to obtain standard compounds with complicated structure. The combination of natural products chemistry and biochemistry is necessary in the conduct of more high-level research on flavonoid biosynthesis. 


\section{Current situation: Delay in the incorporation of natural products chemistry into omics approaches}

In 1999, Veit and Pauli found it surprising that the structures of the phenolics accumulating in A. thaliana had not been studied in detail [70]. Only a few papers described Arabidopsis flavonoids, possibly because researchers in natural products chemistry find studying a non-medicinal plant such as Arabidopsis less beneficial [71-74]. In the genomic era, natural products chemistry is necessary for omics approaches. However, the integration of these 2 methods is a very slow process, for 3 possible reasons: (1) the potential of natural products chemistry in phytochemical genomics is unrecognized; (2) all metabolome elements, i.e., all cell metabolites, are not systematically isolated; and (3) a scientific community effort that combines natural products chemistry and metabolomics or conventional approaches is lacking. For the promotion of the entry of natural products chemistry into omics approaches, the improvements are required below.

\section{Recognition of the potential of natural products chemistry in functional genomics}

Using novel or known compounds can lead to the discovery of structural information, biosynthetic pathways, and the functions of new genes. Such advancements are initiated using natural products chemistry to identify secondary metabolites. Therefore, collaboration between researchers in natural products chemistry and those in modern omics is very important $[9,75]$. 


\section{Systematic isolation approach to metabolomics}

It is believed that $>200,000$ metabolites are involved in plant metabolism. These metabolites have a broad spectrum of chemical diversity and low to high polarity [76, 77]. We have presented a list of the secondary metabolites found in Arabidopsis (Table 2) [52]. It is extremely difficult for one research group to elucidate an entire metabolome by using conventional approaches for compound isolation because of the wide chemical diversity of metabolome. Consequently, systematic approaches should be developed for the targeted isolation of compounds with the same polarity or of the same metabolite group.

AnalytiCon Discovery (http://www.ac-discovery.com/), which commercially supplies secondary metabolites, has established a systematic method for compound isolation. This method includes high-performance liquid chromatography-diode array detection (HPLC-DAD) for profiling, HPLC-evaporative light scattering detection (LC-ELSD) for quantification, LC-MS for structure annotation, automated HPLC for purification, and high-throughput structure identification by using in-house and natural products databases. The structure of unidentified compounds is then elucidated using 1D and 2D nuclear magnetic resonance (NMR) spectroscopy $\left[{ }^{1} \mathrm{H},{ }^{13} \mathrm{C}\right.$, heteronuclear single quantum coherence (HSQC), heteronuclear multiple-bond connectivity (HMBC), and correlation spectroscopy (COSY)] [78]. Using these methods, AnalytiCon Discovery has been a pioneer in providing a vast collection of secondary plant metabolites of plants (3,674 compounds) for many years.

In the academic field, however, manpower is required instead of a 
well-constructed isolation system. Researchers who are skilled at targeted isolation of a particular crude fraction and metabolite group (e.g., flavonoids) can experiment with combinations of individual approaches to overcome the difficulties associated with the chemical diversity of metabolome. The use of several public NMR databases that are currently under development will increase the throughput of identification or structure elucidation (Table 3). It is possible to develop different systematic isolation approaches for the metabolome.

A scientific community effort that combines natural products chemistry and metabolomics or conventional approaches

Organized scientific community efforts that combine transcriptomics and conventional approaches, such as biochemistry and genetics, are quite well established $[79,80]$. Full-length Arabidopsis cDNAs are distributed through the Arabidopsis Biological Resource Center

$(\mathrm{ABRC})$ (http://www.biosci.ohio-state.edu/pcmb/Facilities/abrc/abrchome.htm), which is a publicly available DNA resource. Several resources for obtaining mutants for reverse genetics are provided by a number of organizations [T-DNA-tagged mutants: SALK (http://signal.salk.edu/), SAIL (http://arabidopsis.info/students/paaras/sail.htm), and GABI-Kat (http://www.gabi-kat.de/); transposon-tagged lines: Cold Spring Harbor Laboratory (http://genetrap.cshl.edu/) and RIKEN PSC (http://www.brc.riken.jp/lab/epd/catalog/transposon.html); and activation-tagged lines: RIKEN PSC (http://amber.gsc.riken.jp/act/top.php) and TILLING mutants (http://tilling.fhcrc.org/)] [9]. 
An organized scientific community effort that combines natural products chemistry and metabolomics or conventional approaches for the collection and distribution of standard compounds is also required. Researchers in natural products chemistry, biochemistry, or genetics can use these resources and promote donor-user collaboration for mutual benefit (Fig. 1). Standards can be obtained only by purchase from companies (Table 4), transfer by collaboration, or free transfer. Because it is difficult to discuss the structure of metabolites only based on the information from LC-DAD and molecular ions on mass spectrometry [KNApSAcK (http://kanaya.naist.jp/KNApSAcK/) combined with MS/MS spectral tag (http://prime.psc.riken.jp/) $[50,51,79,80]$, the construction of the science community for exchange and stock of standard compounds is definitely required. 


\section{Future outlook: Use of polyhedral approaches leading to great advancements in plant biotechnology}

Model organisms, including plants, whose entire genomes have been sequenced by next generation sequencers, are increasingly being focused [GOLD (http://genomesonline.org/index2.htm)] [81]. Because of its small genome size, Arabidopsis does not biosynthesize compounds with complex structures and relatively large molecular weights [52]. However, the genome size of model-like plants or crop plants is predicted to be much larger than that of Arabidopsis. The number of structurally complex metabolites with structural isomers will in turn increase according to the genome size [82]. In the future, the use of natural products chemistry in the polyhedral approach will be increasingly useful for identifying these complex metabolites, in functional genomics, and in plant biotechnology. Overcoming the present problems associated with omics approaches will enable us to efficiently use plants for producing secondary metabolites according to our needs [83-85]. 


\section{Acknowledgements}

We extend our apologies to authors whose works have not been cited because of limited space. The authors thank current colleagues and the researchers. The authors also thank Dr. Takayuki Tohge (Max Planck Institute for Molecular Plant Physiology, Germany) and Eriko Hamada-Nakaoka (Wako Pure Chemical Industries, Ltd., Japan) for helpful advices on the manuscript. This work was supported in part by Grants-in-aid from the Ministry of Education, Culture, Sports, Science and Technology, Japan, and CREST, JST. 


\section{References}

1 Newman, D. and Cragg, G. M. (2007) Natural products as sources of new drugs over the last 25 years. J. Nat. Prod. 70, 461-477

2 Arabidopsis genome initiative. (2000) Analysis of the genome sequence of the flowering plant Arabidopsis thaliana. Nature 408, 796-815

3 International Rice Genome Sequencing Project. (2005) The map-based sequence of the rice genome. Nature 436, 793-800

4 Jaillon, O. et al. (2007) The grapevine genome sequence suggests ancestral hexaploidization in major angiosperm phyla. Nature 449, 463-467

5 Ming, R. et al. (2008) The draft genome of the transgenic tropical fruit tree papaya (Carica papaya Linnaeus). Nature 452, 991-996.

6 Tuskan, G. A. et al. (2006) The genome of black cottonwood, Populus trichocarpa (Torr. \& Gray). Science 313, 1596-1604

7 Yu, J. et al. (2002) A draft sequence of the rice genome. (Oryza sativa L. ssp. Indica). Science 296, 79-92

8 Huang, S. et al. (2009) The genome of the cucumber, Cucumis sativus L. Nat. Genet. 41, 1275-1281

9 Yonekura-Sakakibara, K. and Saito, K. (2009) Functional genomics for plant natural product biosynthesis. Nat. Prod. Rep. 26, 1466-1487

10 Schijlen, E. G. et al. (2007) RNAi silencing of chalcone synthase, the first step in the flavonoid biosynthesis pathway, leads to parthenocarpic tomato fruit. 
Plant Physiol. 144, 1520-1530

11 Fernie, A. R. and Schauer, N. (2008) Metabolomics-assisted breeding: a viable option for crop improvement? Trends Genet. 25, 39-48

12 Dixon, R. A. and Paiva, N. L. (1995) Stress-induced phenylpropanoid metabolism. Plant Cell 7, 1085-1097

13 Sirikantaramas, S. et al. (2008) Mutations in topoisomerase I as a self-resistance mechanism coevolved with the production of the anticancer alkaloid camptothecin in plants. Proc. Natl. Acad. Sci. USA 105, 6782-6786

14 Sirikantaramas, S. et al. (2009) A survival strategy: The coevolution of the camptothecin biosynthetic pathway and self-resistance mechanism. Phytochemistry 70, 1894-1898

15 Field, B. and Osbourn, A. E. (2008) Metabolic diversification-Independent assembly of operon-like gene clusters in different plants. Science 320, $543-547$

16 Sudo, H. et al. (2002) Bioreactor production of camptothecin by hairy root cultures of Ophiorrhiza pumila. Biotechnol Lett. 24, 359-363

17 Asano, T. et al. (2004) Camptothecin production by in vitro cultures of Ophiorrhiza liukiuensis and O. kuroiwai. Plant Biotech. 21, 275-281

18 Hirai, M. Y. et al. (2007) Omics-based identification of Arabidopsis Myb transcription factors regulating aliphatic glucosinolate biosynthesis. Proc. Natl. Acad. Sci, USA, 104, 6478-6483

19 Jin, H. et al. (2000) Transcriptional repression by AtMYB4 controls 
production of UV-protecting sunscreen in Arabidopsis. EMBO J. 19, $6150-6161$

20 van der Fits, L. and Memelink, J. (2000) ORCA, a jasmonate-responsive transcriptional regulator of plant primary and secondary metabolism. Science $289,295-297$

21 Memelink, J. et al. (2001) ORCAnization of jasmonate-responsive gene expression in alkaloid metabolism. Trends Plant Sci. 6, 212-219

22 Kato, N. et al. (2007) Identification of a WRKY protein as a transcriptional regulator of benzylisoquinoline alkaloid biosynthesis in Coptis japonica. Plant Cell Physiol. 48, 8-18

23 Yamazaki, Y. et al. (2004) Biosynthesis of camptothecin. In silico and in vivo tracer study from $\left[1-{ }^{13} \mathrm{C}\right]$ glucose. Plant Physiol. $134,161-170$

24 Harborne, J. B. et al. (1999) Phytochemical dictionary: A Handbook of Bioactive compounds from Plants, 2nd ed. (Londong: Taylor \& Francis)

25 Anderson, Ø. M. and Markham, K. R. et al. (2006) Flavonoids: Chemistry, Biochemistry, and Applications. (Boca Raton, FL: CRC Taylor \& Francis)

26 Bais, H. P. et al. (2003) Allelopathy and exotic plant invasion: From molecules and genes to species interactions. Science 301, 1377-1380

27 Bais, H. P. et al. (2004) How plants communicate using the underground information superhighway. Trends Plant Sci. 19, 26-32

28 Field, B. et al. (2006) First encounters - deployment of defence-related natural products by plants. New Phytol. 172, 193-207 
29 Bednarek, P. and Osbourn, A. E. (2009) Plant-microbe interactions: chemical diversity in plant defense. Science 8, 746-748

30 Boue, S. M. et al. (2009) Phytoalexin-enriched functional foods. J. Agric. Food Chem. 57, 2614-2622

31 Nakajima, J. et al. (2004) LC/PDA/ESI-MS profiling and radical scavenging activity of anthocyanins in various berries. J. Biomed. Biotech. 5, 241-247

32 Tohge, T et al. (2005) Enhanced radical scavenging activity of genetically modified Arabidopsis seeds. Biotechnol. Lett. 27, 297-303

33 Butelli, E. et al. (2008) Enrichment of tomato fruit with health-promoting anthocyanins by expression of select transcription factors. Nat. Biotechnol. 26, $1301-1308$

34 Yazaki, K. et al. (2009) Prenylation of aromatic compounds, a key diversification of plant secondary metabolites. Phytochemistry 70, 1739-1745

35 Springob, K. et al. (2003) Recent advances in the biosynthesis and accumulation of anthocyanins. Nat. Prod. Rep. 20, 288-303

36 Grotewold, E. (2008) Transcription factors for predictive plant metabolic engineering: are we there yet? Curr. Opin. Biotechnol. 19, 138-144

37 Koes, R. et al. (2005) Flavonoids: a colorful model for the regulation and evolution of biochemical pathways. Trends Plant Sci. 10, 236-242

38 Tanaka, Y. and Ohmiya, A. (2008) Seeing is believing: engineering anthocyanin and carotenoid biosynthetic pathways. Curr. Opin. Biotechnol. 19, $190-197$ 
39 Hirai, M. Y. et al. (2004) Integration of transcriptomics and metabolomics for understanding of global responses to nutritional stresses in Arabidopsis thaliana. Proc. Natl. Acad. Sci. USA 101, 10205-10210

40 Hirai, M. Y. et al. (2005) Elucidation of gene-to-gene and metabolite-to-gene networks in Arabidopsis by integration of metabolomics and transcriptomics. J. Biol. Chem. 280, 25590-25595

41 Yonekura-Sakakibara, K. et al. (2008) Comprehensive flavonol profiling and transcriptome coexpression analysis leading to decoding gene-metabolite correlations in Arabidopsis. Plant Cell 20, 2160-2176

42 Tohge, T. et al. (2005) Functional genomics by integrated analysis of metabolome and transcriptome of Arabidopsis plants overexpressing an MYB transcription factor. Plant J. 42, 218-235

43 Rischer, H. et al. (2006) Gene-to-metabolite networks for terpenoid indole alkaloid biosynthesis in Catharanthus roseus cells. Proc. Natl. Acad. Sci, USA $103,5614-5619$

44 Nakamura, Y. et al. (2007) Differential metabolomics unraveling light/dark regulation of metabolic activities in Arabidopsis cell culture. Planta 227, $57-66$

45 Malitsky, S. et al. (2008) The transcript and metabolite networks affected by the two clades of Arabidopsis glucosinolate biosynthesis regulators. Plant Physiol. 148, 2021-2049

46 Fukushima, A. et al. (2009) Impact of clock-associated Arabidopsis 
pseudo-response regulators in metabolic coordination. Proc. Natl. Acad. Sci, USA 106, 7251-7256

47 Urano, K. et al. (2009) Characterization of the ABA-regulated global responses to dehydration in Arabidopsis by metabolomics. Plant J. 57, $1065-1078$

48 Maruyama, K. et al. (2009) Metabolic pathways involved in cold acclimation identified by integrated analysis of metabolites and transcripts regulated by DREB1A and DREB2A. Plant Physiol. 150, 1972-1980

49 Okazaki, Y. et al. (2009) A chloroplastic UDP-glucose pyrophosphorylase from Arabidopsis is the committed enzyme for the first step of sulfolipid biosynthesis. Plant Cell 21, 892-909

50 Matsuda, F. et al. (2009) MS/MS spectral tag (MS2T)-based annotation of non-targeted profile of plant secondary metabolites. Plant J. 57, 555-577

51 Matsuda, F. et al. (2010) AtMetExpress development: A phytochemical atlas of Arabidopsis thaliana development. Plant Physiol. 152, 566-578

52 Nakabayashi, R. et al. (2009) Metabolomics-oriented isolation and structure elucidation of 37 compounds including two anthocyanins from Arabidopsis thaliana. Phytochemistry 70, 1017-1029

53 Tohge, T. et al. (2007) Phytochemical genomics in Arabidopsis thaliana: A case study for functional identification of flavonoid biosynthesis genes. Pure Appl. Chem. 79, 811-823

54 Jones, P. et al. (2003) UGT73C6 and UGT78D1 - glycosyltransferases 
involved in flavonols glycoside biosynthesis in Arabidopsis thaliana. J. Biol. Chem. 278, 43910-43918

55 Yonekura-Sakakibara, K. et al. (2007) Identification of a flavonol 7-O-rhamnosyltransferase gene determining flavonoid pattern in Arabidopsis by transcriptome coexpression analysis and reverse genetics. J. Biol. Chem. $282,14932-14941$

56 Fraser, C. M. et al. (2007) Related Arabidopsis serine carboxypeptidase-like sinapoylglucose acyltransferases display distinct but overlapping substrate specificities. Plant Physiol. 144, 1986-1999

57 Luo, J. et al. (2007) Convergent evolution in the BAHD family of acyl transferases: identification and characterization of anthocyanin acyl transferases from Arabidopsis thaliana. Plant J. 50, 678-695

58 Gonzalez, A. et al. (2008) Regulation of the anthocyanin biosynthetic pathway by the TTG1/bHLH/Myb transcriptional complex in Arabidopsis seedlings. Plant J. 53, 814-827

59 Mehrtens, F. et al. (2005) The Arabidopsis transcription factor MYB12 is a flavonol-specific regulator of phenylpropanoids biosynthesis. Plant Physiol. $138,1083-1096$

60 Stracke, R. et al. (2007) Differential regulation of closely related R2R3-MYB transcription factors controls flavonol accumulation in different parts of the Arabidopsis thaliana seedling. Plant J. 50, 660-677

61 Borevitz, J. O. et al. (2000) Activation tagging identifies a conserved MYB 
regulator of phenylpropanoids biosynthesis. Plant Cell 12, 2383-2393

62 Rowan, D. D. et al. (2009) Enviromental regulation of leaf colour in red 35S:PAP1 Arabidopsis thaliana. New Phytol. 182, 102-115

63 Dubos, C. et al. (2009) MYBL2 is a new regulator of flavonoid biosynthesis in Arabidopsis thaliana. Plant J. 55, 940-953

64 Matsui, K. et al. (2009) AtMYBL2, a protein with a single MYB domain, acts as a negative regulator of anthocyanin biosynthesis in Arabidopsis. Plant J. 55, 954-967

65 Zimmermann, P. et al. (2004) GENEVESTIGATOR. Arabidopsis microarray database and analysis toolbox. Plant Physiol. 136, 2621-2632

66 Zimmermann, P. et al. (2005) Gene-expression analysis and network discovery using Genevestigator. Trends Plant Sci. 10, 407-409

67 Obayashi, T. et al. (2007) ATTED-II: a databese of co-expressed genes and cis elements for identifying co-regulated gene groups in Arabidopsis. Nucleic Acids Res. 35, D863-D869

68 Goda, H. et al. (2008) The AtGenExpress hormone and chemical treatment data set: experimental design, data evaluation, model data analysis and data access. Plant J. 55, 526-542

69 Saito, K. et al. (2008) Decoding genes with coexpression networks and metabolomics - 'majority report by precogs'. Trends Plant Sci. 13, 36-43

70 Veit, M. and Pauli, G. F. (1999) Major flavonoids from Arabidopsis thaliana leaves. J. Nat. Prod. 62, 1301-1303 
71 Graham, T. L. (1998) Flavonoid and flavonol glycoside metabolism in Arabidopsis. Plant Physiol. Biochem. 36, 135-144

72 Bloor, S. J. and Abrahams, S. (2002) The structure of the major anthocyanin in Arabidopsis thaliana. Phytochemistry 59, 343-346

73 Routaboul, J. et al. (2006) Flavonoid diversity and biosynthesis in seed of Arabidopsis thaliana. Planta 224, 96-107

74 Böttcher, C. et al. (2008) Metabolome analisis of biosynthetic mutants reveals a diversity of metabolic changes and allows identification of a large number of new compounds in Arabidopsis. Plant Physiol. 147, 2107-2120

75 Oksman-Caldentey, KM and Saito, K. (2005) Integrating genomics and metabolomics for engineering plant metabolic pathways. Curr. Opin. Biotechnol. 16, 174-179

76 Dixon, R. A. and Strack, D. (2003) Phytochemistry meets genome analysis, and beyond......... Phytochemistry 62, 815-816

77 D'Auria, J. C. and Gershenzon, J. (2005) The secondary metabolism of Arabidopsis thaliana: growing like a weed. Curr. Opin. Plant Biol. 8, $308-316$

78 Bindseil, K. U. et al. (2001) Pure compound libraries; a new perspective for natural product based drug discovery. Drug Discov. Today 6, 840-847

79 Tohge, T. and Fernie, A. R. (2009) Web-based resources for mass-spectrometry-based metabolomics: a user's guide. Phytochemistry 70, $450-456$ 
80 Brady, S. M. and Provart, N. J. (2009) Web-queryable large-scale data sets for hypothesis generation in plant biology. Plant Cell 21, 1034-1051

81 Ansorge, W. J. (2009) Next-generation DNA sequencing techniques. New Biotechnol. 25, 195-203

82 Kitajima, M. (2007) Chemical studies on monoterpenoid indole alkaloids from medicinal plant resources Gelsemium and Ophiorrhiza. J. Nat. Med. 61, $14-23$

83 Luo, J. et al. (2008) AtMYB12 regulates caffeoyl quinic acid and flavonol synthesis in tomato: expression in fruit results in very high levels of both types of polyphenol. Plant J. 56, 316-326

84 Seki, H. et al. (2008) Licorice $\beta$-amyrin 11-oxidase, a cytochrome P450 with a key role in the biosynthesis of the triterpene sweetener glycyrrhizin. Proc. Natl. Acad. Sci. USA 105, 14204-14209

85 Saito, K. and Matsuda, F. (2010) Metabolomics for functional genomics, systems biology, and biotechnology. Annu. Rev. Plant Biol. 61, in press. 


\section{Figure legends}

Figure 1. A polyhedral approach for understanding of biosynthetic mechanisms of secondary metabolites

The combination of omics and conventional approach could be regarded as omics-guided fusion approach and that of omics-guided fusion approaches could be regarded as a polyhedral approach.

Figure 2. A proposed flavonoid biosynthetic pathway.

The biosynthesis of flavonol glycoside has almost characterized $[41,42,54,55]$. ' $\mathrm{f}$ number' and 'A number' compounds were annotated and partly identified (Table 1) [41, 42]. Red arrows indicate in vitro malonyltransferase reactions with substrates (A3, A6, A7 and A10) [57]. The thickest arrow shows the most efficient reaction.

CHS; chalcon synthase, CHI; chalcon isomerase, F3H; flavanone 3-hydroxylase, F3'H; flavonoid 3'-hydroxylase, FLS1; flavonol synthase1, OMT1; caffeic acid/5-hydroxyferulic acid $O$-methyltransferase, flavonol 3'-O-methyltransferase, Fd3GlcT; flavonoid 3-O-glucosyltransferase, F3AraT; flavonol 3-O-arabinosyltransferase, F3RhaT; flavonol 3-O-rhamnosyltransferase, F7RhaT; flavonol 7-O-rhamnosyltransferase, F7GlcT; flavonol 7-O-glucosyltransferase, XylT: xylosyltransferase $\quad$ (unknown), $\quad p$-CouT; $\quad$ Anthocyanin 3-O-glucoside:6"-O-p-coumaroyltransferase, GlcT; glucosyltransferase (unknown), 5GlcT; Anthocyanin 5-O-glucosyltransferase, MaT; Anthocyanin 
5-O-glucoside:6"'-O-malonyltransferase, $\quad$ SAT; $\quad$ sinapoylglucose:anthoacyanin acyltransferase.

Cy; cyanidin, ia; intermidiate of anthocyanin of A1 to A11, ia1; cyanidin 5-O- $\beta$-D-glucopyranoside, ia2; cyanidin 5-O-[6-O-(malonyl)- $\beta$-D-glucopyranoside], ia3; cyanidin 3-O- $\beta$-D-glucopyranoside, $\quad$ ia4; cyanidin 3-O- $\beta$-D-glucopyranoside-5-O- $\beta$-D-glucopyranoside, $\quad$ ia5; cyanidin 3-O- $\beta$-D-glucopyranoside-5-O-[6-O-(malonyl)- $\beta$-D-glucopyranoside $], \quad$ ia6; cyanidin 3-O-[2-O-( $\beta$-D-xylopyranosyl)- $\beta$-D-glucopyranoside $], \quad$ ia $7 ; \quad$ cyanidin 3-O-[2-O-(2-O-E-sinapoyl- $\beta$-D-xylopyranosyl)- $\beta$-D-glucopyranoside], ia8; cyanidin 3-O-[2-O-(2-O-(E-sinapoyl- $\beta$-D-xylopyranosyl)-5- $O-[6-O-($ malonyl $)-\beta-\mathrm{D}-\mathrm{glucopyranosi}$ de], $\quad$ ia9; $\quad$ cyanidin 3-O-[2-O-( $\beta$-D-xylopyranosyl)-6-O-(E-p-coumaroyl)- $\beta$-D-glucopyranoside $], \quad$ ia10; cyanidin 3-O-[2-O-(2-O-E-sinapoyl- $\beta$-D-xylopyranosyl)-6- $O$-(E-p-coumaroyl)- $\beta$-D-glucopyranos ide], ial1; cyanidin 3-O-[2-O-( $\beta$-D-xylopyranosyl)-6-O-(4-O-( $\beta$-D-glucopyranosyl- $E$ - $p$-coumaroyl $)-\beta$-D-glu copyranoside), ia12; cyanidin 3-O-[2-O-(2-O-E-sinapoyl- $\beta$-D-xylopyranosyl)-6-O-(4-O-( $\beta$-D-glucopyranosyl)-E-p-co umaroyl)- $\beta$-D-glucopyranoside]. 
Challenge to understanding of biosynthetic mechanism

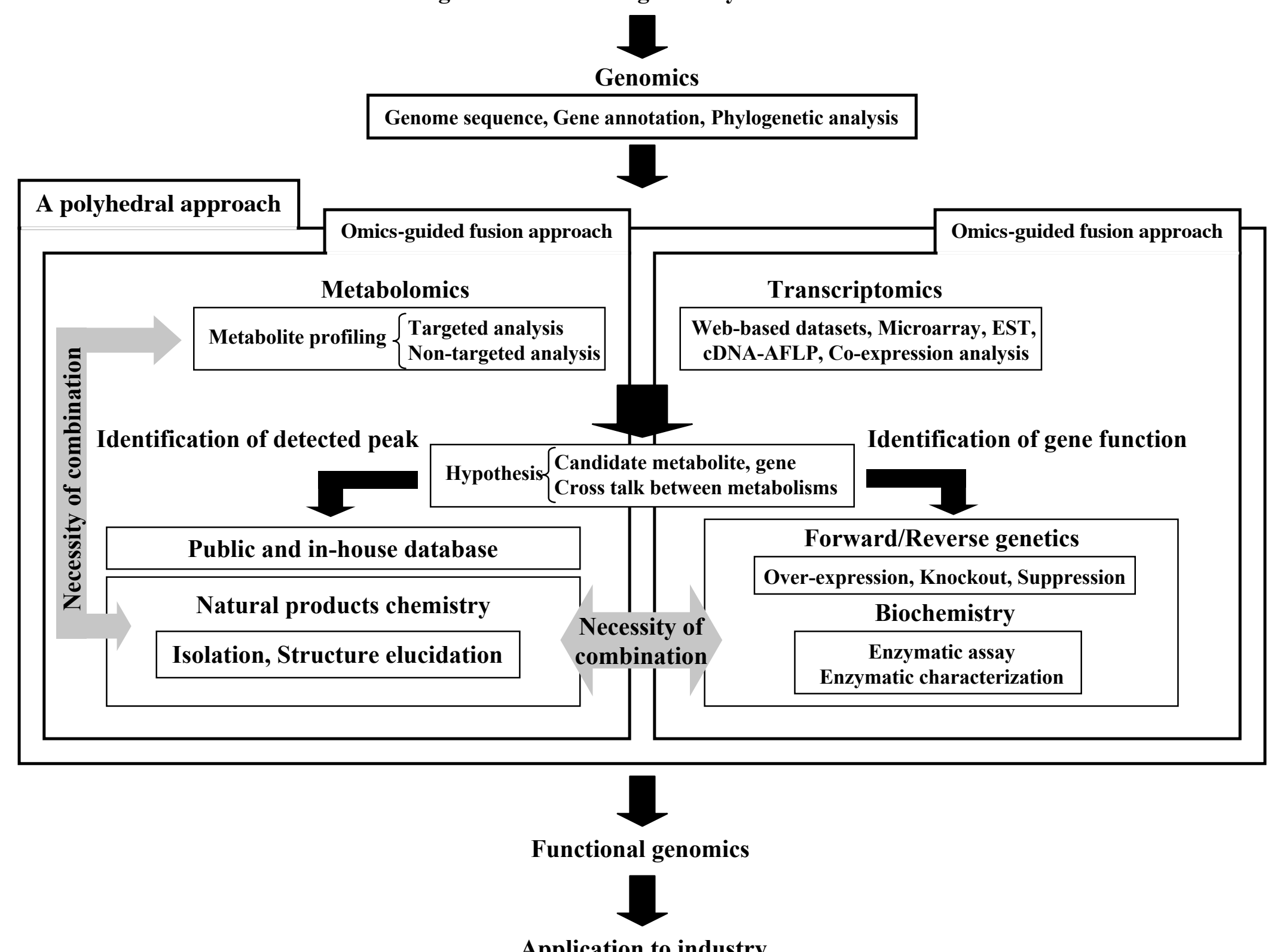

Application to industry 
Flavonol-glycoside biosynthetic pathway

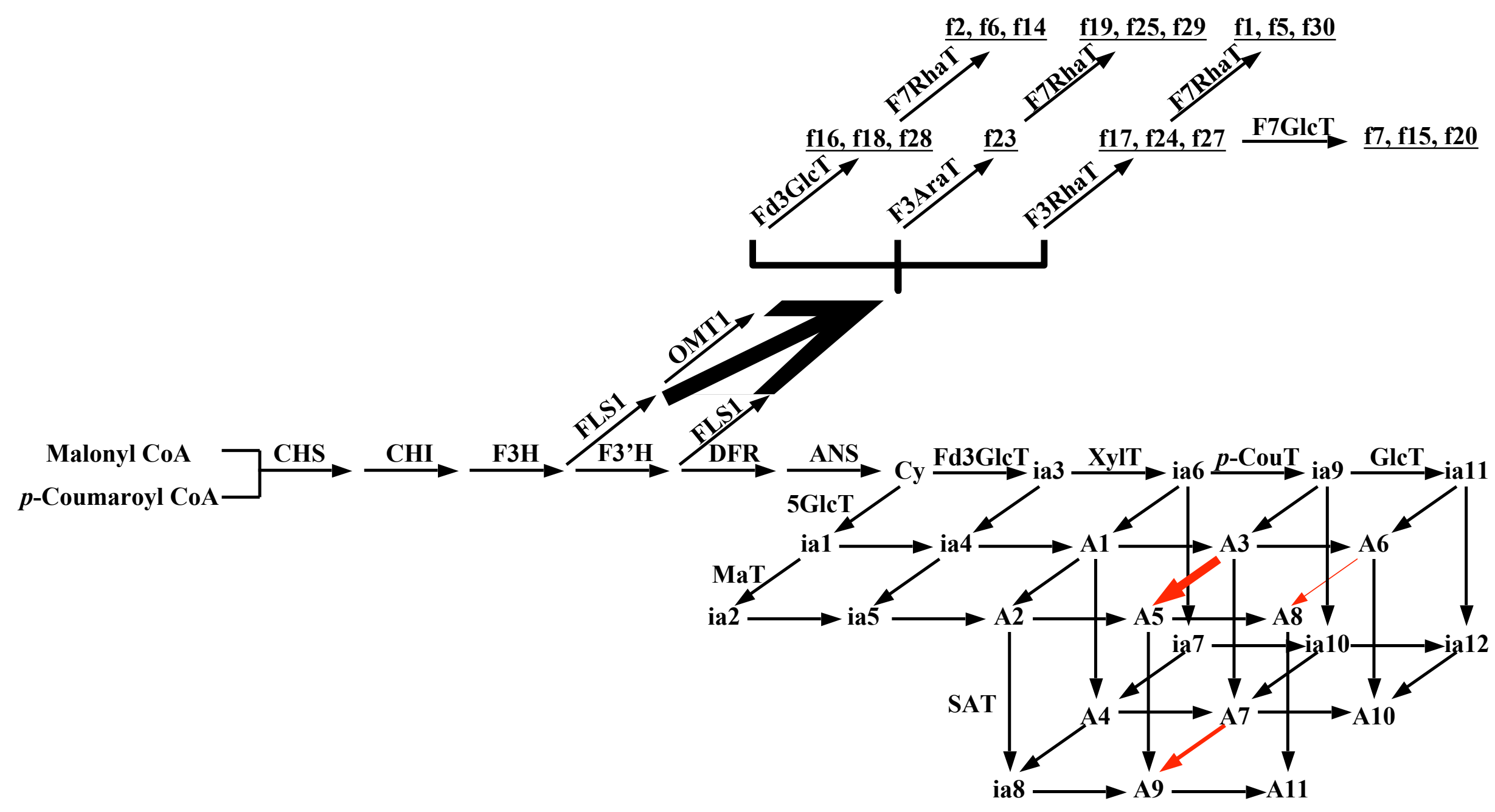

Anthocyanin biosynthetic pathway 
TABLE 1. Arabidopsis flavonoids

\begin{tabular}{|c|c|c|c|c|c|c|c|c|c|}
\hline Flavono & & $\mathrm{R}_{1}$ & $\mathrm{R}_{2}$ & $\mathrm{R}_{3}$ & Anthocyanin & & $R_{1}$ & $\mathrm{R}_{2}$ & $\mathrm{R}_{3}$ \\
\hline f1 & 0 & $\mathrm{H}$ & rhamnoside & rhamnoside & A1 & $\triangle$ & $\mathrm{H}$ & $\mathrm{H}$ & $\mathrm{H}$ \\
\hline f2 & 0 & $\mathrm{H}$ & glucoside & rhamnoside & A2 & & $\mathrm{H}$ & $\mathrm{H}$ & malonyl \\
\hline f3 & O & $\mathrm{H}$ & rhamnosyl $(1 \rightarrow 2)$ glucoside & rhamnoside & A3 & 0 & $\mathrm{H}$ & $p$-coumaroyl & $\mathrm{H}$ \\
\hline $\mathrm{f} 4$ & & $\mathrm{H}$ & glucosyl $(1 \rightarrow 6)$ glucoside & rhamnoside & A4 & & $\mathrm{H}$ & $p$-coumaroyl-glucose & $\mathrm{H}$ \\
\hline f5 & O & $\mathrm{OH}$ & rhamnoside & rhamnoside & A5 & 0 & sinapoyl & $p$-coumaroyl & $\mathrm{H}$ \\
\hline f6 & O & $\mathrm{OH}$ & glucoside & rhamnoside & A6 & 0 & $\mathrm{H}$ & $p$-coumaroyl-glucose & malonyl \\
\hline $\mathrm{f} 7$ & $\triangle$ & $\mathrm{OH}$ & rhamnoside & glucoside & A7 & & sinapoyl & $p$-coumaroyl & $\mathrm{H}$ \\
\hline f8 & O & $\mathrm{OH}$ & rhamnosyl $(1 \rightarrow 2)$ glucoside & rhamnoside & A8 & $\bigcirc$ & $\mathrm{H}$ & $p$-coumaroyl-glucose & malonyl \\
\hline f9 & & $\mathrm{H}$ & conjugate & & A9 & $\mathrm{O}$ & sinapoyl & $p$-coumaroyl & malonyl \\
\hline f10 & & $\mathrm{H}$ & hexose-deoxyhexose & - & A10 & 0 & sinapoyl & $p$-coumaroyl-glucose & $\mathrm{H}$ \\
\hline f11 & & $\mathrm{H}$ & conjugate & & A11 & 0 & sinapoyl & $p$-coumaroyl-glucose & malonyl \\
\hline $\mathrm{f} 12$ & & $\mathrm{H}$ & rhamnoside conjugate & - & & & & & \\
\hline $\mathrm{f} 13$ & & $\mathrm{OH}$ & hexosyl deoxyhexose & - & Flavonol & & & & \\
\hline f14 & & $\mathrm{OCH}_{3}$ & glucoside & rhamnoside & & & & & \\
\hline f15 & & $\mathrm{OCH}_{3}$ & rhamnoside & glucoside & & & & & \\
\hline f16 & $\triangle$ & $\mathrm{OH}$ & glucoside & $\mathrm{H}$ & & & & & \\
\hline f17 & $\triangle$ & $\mathrm{H}$ & rhamnoside & $\mathrm{H}$ & & & & & \\
\hline f18 & $\triangle$ & $\mathrm{H}$ & glucoside & $\mathrm{H}$ & & & & & \\
\hline f19 & $\square$ & $\mathrm{H}$ & arabinoside & rhamnoside & & & & $\mathbf{R}_{2}$ & \\
\hline $\mathrm{f} 20$ & & $\mathrm{H}$ & rhamnoside & glucoside & & & & & \\
\hline $\mathrm{f} 21$ & & $\mathrm{H}$ & hexose glucoside & rhamnoside & & & & & \\
\hline $\mathrm{f} 22$ & & $\mathrm{H}$ & pentose, deoxyhexose & rhamnoside & Anthocyanin & & & & \\
\hline $\mathrm{f} 23$ & & $\mathrm{OH}$ & arabinoside & $\mathrm{H}$ & & & & & \\
\hline $\mathrm{f} 24$ & O & $\mathrm{OH}$ & rhamnoside & $\mathrm{H}$ & & & & & \\
\hline $\mathrm{f} 25$ & $\square$ & $\mathrm{OH}$ & arabinoside & rhamnoside & & & & & \\
\hline $\mathrm{f} 26$ & & $\mathrm{OH}$ & glucose, hexose & - & & & & & \\
\hline f27 & & $\mathrm{OCH}_{3}$ & rhamnoside & $\mathrm{H}$ & & & & & \\
\hline $\mathrm{f} 28$ & $\triangle$ & $\mathrm{OCH}_{3}$ & glucoside & H & & & & & \\
\hline f29 & & $\mathrm{OCH}_{3}$ & arabinoside & rhamnoside & & & & & \\
\hline $\mathrm{f} 30$ & & $\mathrm{OCH}_{3}$ & rhamnoside & rhamnoside & & & & & \\
\hline $\begin{array}{l}f 31 \\
f 32\end{array}$ & & $\begin{array}{l}\mathrm{OCH}_{3} \\
\mathrm{OCH}_{3}\end{array}$ & $\begin{array}{c}\text { glucose, hexose } \\
\text { rhamnosyl }(1 \rightarrow 2) \text { glucoside }\end{array}$ & $\stackrel{-}{\text { rhamnoside }}$ & & & & $\begin{array}{l}\mathrm{HO}^{\mathrm{H}} \\
\mathrm{OH}\end{array}$ & \\
\hline
\end{tabular}

$\bigcirc$ : Isolated standard from Arabidopsis, $\triangle$ : Purchased standard from company, $\square$ : standard synthesized by enzymatic reaction 
TABLE 2. Actual and expected distribution of Arabidopsis metabolites.

\begin{tabular}{cl}
\hline Fraction & \multicolumn{1}{c}{ Group } \\
\hline$n-\mathrm{Hexane}$ & Fatty acid, Terpenoid \\
EtOAc & Apocarotenoid, Carotenoid, Chlorophyll, Flavonol (mono and di)glycoside, Galactolipid, \\
& Indole, Phenylpropanoid \\
$n-\mathrm{BuOH}$ & Acylated anthocyanin, Flavonol (di and tri)glycoside, Phenylpropanoid \\
$\mathrm{H}_{2} \mathrm{O}$ & Amino acid, Flavonol triglycoside, Glucosinolate, Nucleoside, Phenylpropanoid, Sugar \\
\hline
\end{tabular}


TABLE 3. Useful databases for structure elucidation

Database URL

Chemical-shift-based prediction

Biological Magnetic Resonance Data Bank

C-13 NMR Chemical Shifts

\section{NMR Data Libraries}

NMRshiftDB

Proton NMR Chemical Shift

\section{SDBS}

Spectral Similarity Search

SpinAssign

\section{Structure-based prediction}

\section{CSEARCHlite}

NMR Database of Lignin and Cell Wall Model Compounds nmrdb.org

NPEdia Compounds Search

Web Version of SciFinder http://www.bmrb.wisc.edu/metabolomics/query_metab.php http://www.chem.wisc.edu/areas/reich/Handouts/nmr-c13/cdata.htm http://www.acdlabs.com/products/spec_lab/predict_nmr/data_libraries.html http://www.ebi.ac.uk/nmrshiftdb/

http://www.chem.wisc.edu/areas/reich/Handouts/nmr-h/hdata.htm http://riodb01.ibase.aist.go.jp/sdbs/cgi-bin/direct_frame_top.cgi http://nmrpredict.orc.univie.ac.at/case/propose.php http://prime.psc.riken.jp/?action=nmr_search

http://nmrpredict.orc.univie.ac.at/csearchlite/index.php http://ars.usda.gov/Services/docs.htm?docid=10491 http://www.nmrdb.org/predictor?smiles $=0 \% 3 \mathrm{dC} 4 \mathrm{c} 5 \mathrm{c}$ http://npd.riken.jp/npedia/keywords.php http://www.cas.org/products/scifindr/sfweb/ 
TABLE 4. Companies provide standards of secondary metabolites

$$
\text { Company }
$$

URL

Advanced Technology \& Industrial Co, Ltd http://www.advtechind.com/

Analyticon Discovery

Apin Chemicals Ltd.

http://www.ac-discovery.com/

ChromaDex, Inc.

http://www.apinchemicals.com/

Herb Standard Company

http://www.chromadex.com/index2.html

http://herbstandard.com/

PhytoLab

http://www.phytolab.com/phytolab/

Polyphenols

http://www.polyphenols.com/

TOKIWA PHYTOCHEMICAL CO, LTD

http://www.tokiwaph.co.jp/en/

TransMIT

http://www.transmit.de/en/index.htm 